\title{
Competition between anthocyanin and kaempferol glycosides biosynthesis affects pollen tube growth and seed set of Malus
}

Weifeng Chen ${ }^{1}$, Zhengcao Xiao ${ }^{1,2}$, Yule Wang ${ }^{1}$, Jinxiao Wang ${ }^{1}$, Rui Zhai ${ }^{1}$, Kui Lin-Wang ${ }^{3}$, Richard Espley $\mathbb{B}^{3}$, Fengwang $\mathrm{Ma}^{1}$ and Pengmin $\mathrm{Li}^{1}$

\begin{abstract}
Flavonoids play important roles in regulating plant growth and development. In this study, three kaempferol 3-Oglycosides were identified and mainly accumulated in flowers but not in leaves or fruits of Malus. In Malus, flower petal color is normally white, but some genotypes have red flowers containing anthocyanin. Anthocyanin biosynthesis appears to be in competition with kaempferol 3-O-glycosides production and controlled by the biosynthetic genes. The white flower Malus genotypes had better-developed seeds than the red flower genotypes. In flowers, the overexpression of MYB10 in Malus domestica enhanced the accumulation of anthocyanin, but decreased that of kaempferol 3-O-glycosides. After pollination the transgenic plants showed slower pollen tube growth and fewer developed seeds. Exogenous application of different flavonoid compounds suggested that kaempferol 3-O-glycosides, especially kaempferol 3-O-rhamnoside, regulated pollen tube growth and seed set rather than cyanidin or quercetin 3O-glycosides. It was found that kaempferol 3-O-rhamnoside might regulate pollen tube growth through effects on auxin, the Rho of plants (ROP) GTPases, calcium and the phosphoinositides signaling pathway. With the inhibition of auxin transport, the transcription levels of Heat Shock Proteins (HSPs) and ROP GTPases were downregulated while the levels were not changed or even enhanced when blocking calcium signaling, suggesting that HSPs and ROP GTPases were downstream of auxin signaling, but upstream of calcium signaling. In summary, kaempferol glycoside concentrations in pistils correlated with auxin transport, the transcription of HSPs and ROP GTPases, and calcium signaling in pollen tubes, culminating in changes to pollen tube growth and seed set.
\end{abstract}

\section{Introduction}

Flavonoids are common secondary metabolites with more than 8000 forms discovered in plants. The flavonoids contain six major classes including chalcones, flavones, isoflavonoids, flavanones, flavonols, and anthocyanins ${ }^{1}$. Flavonoid biosynthesis begins with the substrate 4-coumaroyl-CoA, which is stepwise catalyzed by chalcone

\footnotetext{
Correspondence: Pengmin Li (Lipm@nwafu.edu.cn)

${ }^{1}$ State Key Laboratory of Crop Stress Biology for Arid Areas/Shaanxi Key Laboratory of Apple, College of Horticulture, Northwest A\&F University, Yangling, Shaanxi 712100, China

${ }^{2}$ College of Food Science and Technology, Northwest University, Xi'an, Shaanxi 710069, China

Full list of author information is available at the end of the article

These authors contributed equally: Weifeng Chen, Zhengcao Xiao.
}

synthase (CHS) and chalcone flavanone isomerase $(\mathrm{CHI})$ to produce naringenin. Naringenin can be hydroxylated at the $3^{\prime}$-position of the B-ring by flavonoid $3^{\prime}$-hydroxylase $\left(F 3^{\prime} \mathrm{H}\right)$ to produce eriodictyol or hydroxylated at the 3-position of the $\mathrm{C}$-ring by flavanone 3-hydroxylase $(\mathrm{F} 3 \mathrm{H})$ to produce dihydrokaempferol. Eriodictyol or dihydrokaempferol can be further hydroxylated at the 3-position of the $\mathrm{C}$-ring or at the $3^{\prime}$-position of the B-ring by $\mathrm{F} 3 \mathrm{H}$ or $\mathrm{F}^{\prime} \mathrm{H}$ to produce dihydroquercetin $^{2,3}$. In some plants, dihydrokaempferol can also be hydroxylated at both the $3^{\prime}$ - and $5^{\prime}$-positions of the B-ring by flavonoid $3^{\prime} 5^{\prime}$-hydroxylase to produce dihydromyricetin $^{4,5}$. Dihydrokaempferol, dihydroquercetin, and dihydromyricetin are catalyzed by flavonol synthase (FLS) to produce kaempferol, quercetin, and myricetin, respectively,

\section{(c) The Author(s) 2021}

(c) Open Access This article is licensed under a Creative Commons Attribution 4.0 International License, which permits use, sharing, adaptation, distribution and reproduction c. in any medium or format, as long as you give appropriate credit to the original author(s) and the source, provide a link to the Creative Commons license, and indicate if changes were made. The images or other third party material in this article are included in the article's Creative Commons license, unless indicated otherwise in a credit line to the material. If material is not included in the article's Creative Commons license and your intended use is not permitted by statutory regulation or exceeds the permitted use, you will need to obtain permission directly from the copyright holder. To view a copy of this license, visit http://creativecommons.org/licenses/by/4.0/. 
or they are catalyzed by dihydroflavonol 4-reductase (DFR) and anthocyanin synthase (ANS) to produce anthocyanidins $^{4-7}$. Since the aglycones (kaempferol, quercetin, myricetin, and anthocyanidin) are not stable in plants, they are usually glycosylated by the catalysis of UDP-glycose: flavonoid glycosyltransferase (UGT) and then transported and stored in vacuoles. This suggests that there is likely to be competition for the same substrates for the synthesis of different kinds of flavonoid end products ${ }^{8-10}$. For some plants, the variation in flower color is determined by competition between the synthesis of flavonols and anthocyanins $\mathrm{s}^{7,11}$.

Besides affecting color, flavonoids have other important biological functions in plants. The inhibition of flavonoid synthesis by silencing MdCHS or MdUGT88F1 in apple plants resulted in shortened internode lengths, smaller leaves, and a greatly reduced growth rate ${ }^{12,13}$. In the flowers of Petunia, the UV absorbance due to the presence of flavonols plays an important role in attracting pollinators with corresponding flower signal preferences ${ }^{7}$. It was also found that flavonols can modulate stomata aperture behavior ${ }^{14,15}$, root development and gravitropism ${ }^{16-18}$, leaf and trichome development in Arabidopsis ${ }^{19,20}$, self-compatibility in Brassicaceae $^{21}$, and protect plants against pathogens ${ }^{22}$.

Recently, Muhlemann et al. reported that flavonol might control pollen tube growth and integrity by regulating ROS homeostasis in tomato under high-temperature stress ${ }^{23}$. The anthocyanin reduced (are) tomato mutant, which lacks a functional F3H enzyme, has reduced flavonol accumulation in pollen grains and tubes, and produced fewer pollen grains as well as having impaired pollen viability, germination, tube growth, and integrity resulting in reduced seed set $^{23}$. The mutation of $\mathrm{FHH}$ might also decrease the flavonol and anthocyanin accumulation in other flower tissues such as the pistil. However, whether the flavonols in the pistil affected pollen germination and tube growth was not clarified. Moreover, the precise nature of the flavonol compounds in pollen that are involved in this regulation of pollen development was not confirmed.

Of the numerous variety of flavonol compounds in plants, the common major flavonol aglycones are quercetin and kaempferol, with their derivatives being more abundant. The derivatives of these different aglycones might play different roles in plants. For instance, quercetin rather than kaempferol derivatives were shown to modulate basipetal root auxin transport, gravitropism, and elongation in Arabidopsis $^{18}$. In addition, for any flavonol aglycone the different types of UDP-sugars and the position and amount of glycosylation, the glycosylated version might show different functions. For example, it was found that it was specifically kaempferol 3-O-rhamnoside-7-O-rhamnoside, as opposed to any other kaempferol glycoside compounds, that acted as an endogenous polar auxin transport inhibitor in Arabidopsis shoots ${ }^{24}$. Phloretin and its derivatives, a unique group of flavonoids in Malus, showed different antioxidant capacities, even when the chemical structures of each compound simply differed in one hydroxyl group or glycosylation ${ }^{25,26}$. So, to clarify the precise relationships between flavonoid structures and their physiological functions on the pollens, further studies are required.

For Malus, domesticated apple cultivars provide fresh apple fruits or juice for consumption, whereas ornamental crabapple cultivars are widely used in landscaping for their colorful flowers and, largely inedible, fruits. Both the domestic and ornamental cultivars originally derive from the wild species of Malus through different routes of domestication, and the different genotypes of wild species come from the evolution of Malus ${ }^{27,28}$. The evolution process differs from domestication. For instance, the traits considered beneficial by human beings such as fruit size, texture, flavor, and other fruit quality-related traits of Malus, were selected for improvement during domestication ${ }^{28,29}$, whereas the positive traits that contribute to plant survival are likely to be retained and enhanced during Malus evolution. Although positive traits that contribute to plant survival might be lost or abated during domestication, this does not necessarily compromise the cultivar. For instance, in some cultivars, positive traits related to seed development may be lost, but reproduction is still feasible through grafting or other asexual ways. However, for wild species that need to survive in natural conditions, long-term survival may be threatened under the pressure of natural selection if such traits were lost.

To date, a large number of Malus ornamental or edible red flower/flesh cultivars have been successfully developed or are being developed in breeding programs. However, for wild species of Malus, the majority of them have white flowers/flesh (some with pink bud). So, the question arises as to whether flower color is in some way disadvantageous and/or relates to the reproductive capacity of Malus. This is possibly why the wild species of Malus seldom have red flowers. In this study, we used different flower color genotypes of Malus to investigate the relationship between flower color and seed set, an important trait for sexual reproduction capacity. In particular, we focused on the association of flavonoid metabolism in pistils and on pollen tube growth and seed set.

\section{Results}

\section{Correlation between flower color and seed set}

Fourteen genotypes of Malus, including eight white (pink) flower (WF) and six red flower (RF) genotypes, were collected to assess the seed set of fruits (Fig. 1a). Interestingly, it was found that for WF genotypes all seed carpels were full, while for RF genotypes some empty seed carpels were found. Moreover, the numbers of well-developed seeds were significantly fewer in RF genotypes than that in WF genotypes (Fig. 1b). In previous studies, it was shown that 

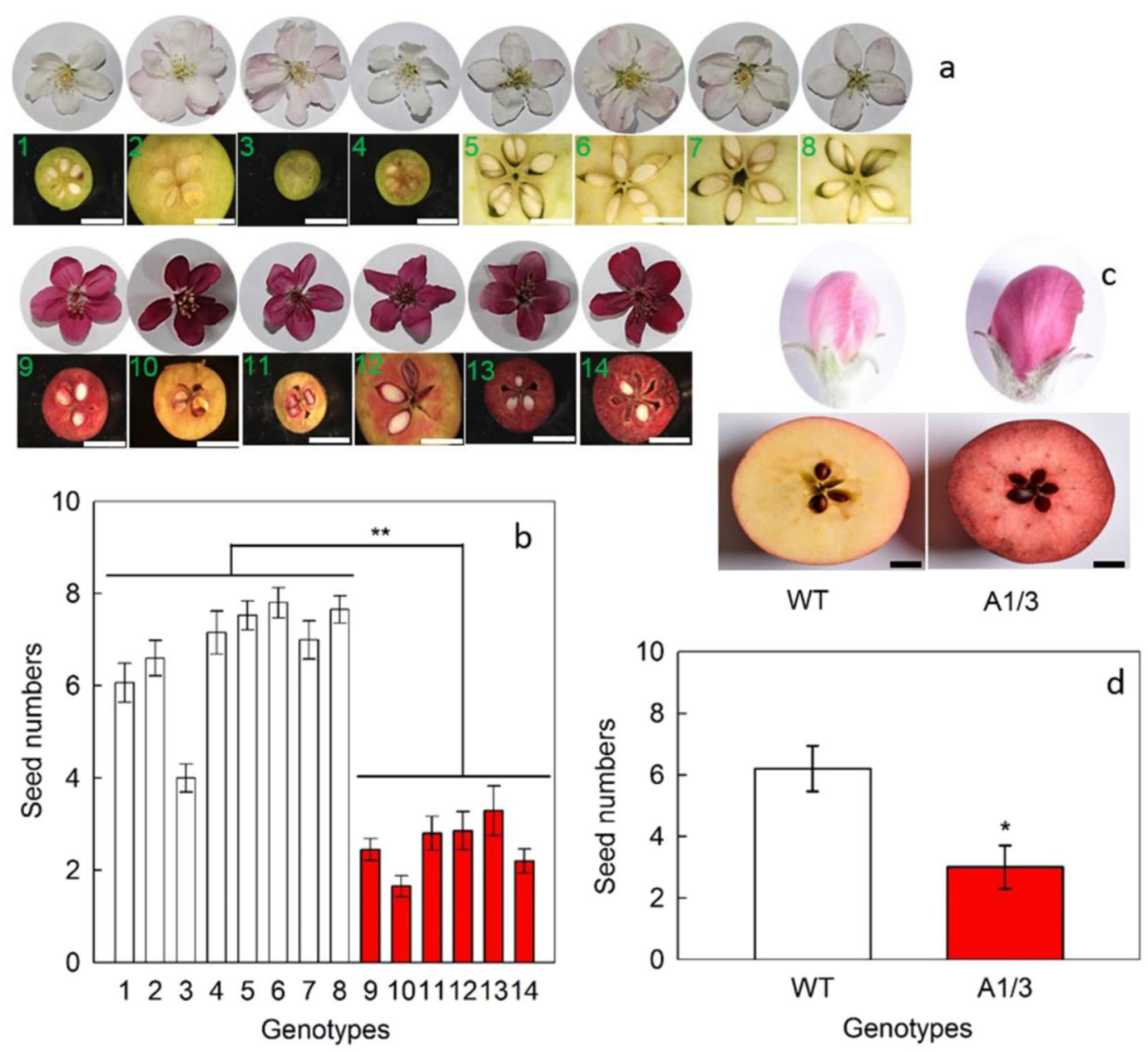

Fig. 1 The phenotypes and seed numbers of different genotypes of Malus plants. $\mathbf{a}$, $\mathbf{b}$ White flower genotypes: 1, M. hupehensis; $2, M$. micromalus; 3, M. halliana; 4, M. baccata; 5, M. domestica 'Golden Delicious'; 6, M. domestica 'Fuji'; 7, M. domestica 'Red Delicious'; 8, M. domestica 'Gala'; and red flower genotypes; 9, M. 'Sparkler'; 10, M. 'Radiant'; 11, M. 'Adams'; 12, M. 'Kelsey'; 13, M. 'Perfect Purple'; 14, M. 'Royalty'. c, d WT, wild type of M. domestica 'Royal Gala'; A1/3, transgenic line with MdMYB10 overexpression of 'Royal Gala'. a, c Bars $=1 \mathrm{~cm}$. b, d White bars represent white flower genotypes and red bars represent red flower genotypes; data are presented as mean $\pm \mathrm{SE}(n=5)$; "*" and "**" mean significant difference between the white flower and the red flower genotypes at $P<0.05$ and at $P<0.01$, respectively, $t$-test

overexpression of $M Y B 10$, a key transcript factor that regulates anthocyanin synthesis in apple, could strongly elevate the anthocyanin content of apple plants ${ }^{30,31}$. In this study, the transgenic apple plants with MYB10 overexpression (RF) and its wild type $M$. domestica 'Royal Gala' (WF), was also used to test the correlation between flower color and seed set. It was found that the transgenic fruits had significantly fewer seeds compared with the wild type (WT) (Fig. 1c, d).

\section{Identification of kaempferol 3-O-glycosides}

The chromatograms of flower flavonoids showed that the transgenic lines (A1/3, A4) of 'Royal Gala' accumulated more anthocyanin (cyanidin 3-O-galactoside), but less of the three unknown flavonoid compounds (K1, K2, and K3) compared with the WT (Fig. 2a). Similarly, the RF genotypes also showed significantly lower amounts of the three unknown compounds compared with the WF genotypes.

To identify the three unknown flavonoid compounds, $5 \mathrm{~kg}$ of M. domestica 'Fuji' flowers was used to isolate the compounds with a purity higher than $98 \%$, and then ESIMS, ${ }^{1} \mathrm{H}$ NMR, and ${ }^{13} \mathrm{C}$ NMR were used to analyze the structures of the three compounds (Fig. 2b, c, Table S1, Fig. S1).

The ESI-MS analysis showed that both $\mathrm{K} 1$ and $\mathrm{K} 2$ had a quasi-molecule ion at $m / z 419.1[\mathrm{M}+\mathrm{H}]^{+}$, while $\mathrm{K} 3$ had a quasi-molecule ion at $m / z 433.2[\mathrm{M}+\mathrm{H}]^{+}$. However, all of them had a fragment at $m / z 287.1$, consistent with the molecular weight of kaempferol in positive mode (Fig. 2c). The fragments indicate a loss of a pentoside moiety for $\mathrm{K} 1$ and $\mathrm{K} 2$, and a loss of the rhamnoside moiety for $\mathrm{K} 3$. Combined with the ${ }^{1} \mathrm{H}$ and ${ }^{13} \mathrm{C}$ NMR analysis results 
a

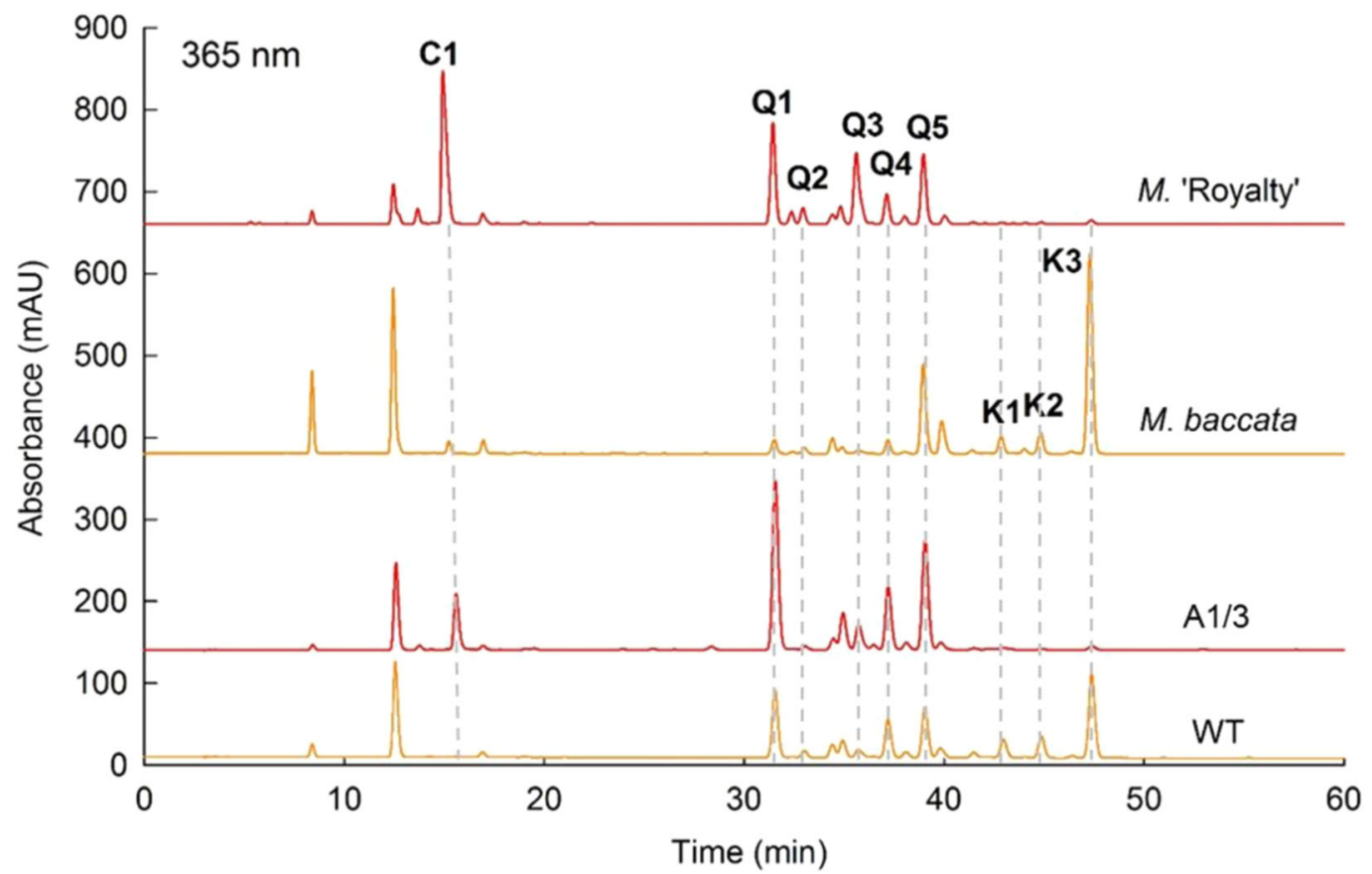<smiles>O=c1c(OC2OC[C@@H](O)[C@H](O)[C@H]2O)c(-c2ccc(O)cc2)oc2cc(O)cc(O)c12</smiles><smiles>O=c1c(OC2OC(CO)C(O)C2O)c(-c2ccc(O)cc2)oc2cc(O)cc(O)c12</smiles><smiles>C[C@H]1O[C@H](Oc2c(-c3ccc(O)cc3)oc3cc(O)cc(O)c3c2=O)[C@H](O)[C@@H](O)[C@@H]1O</smiles>

C
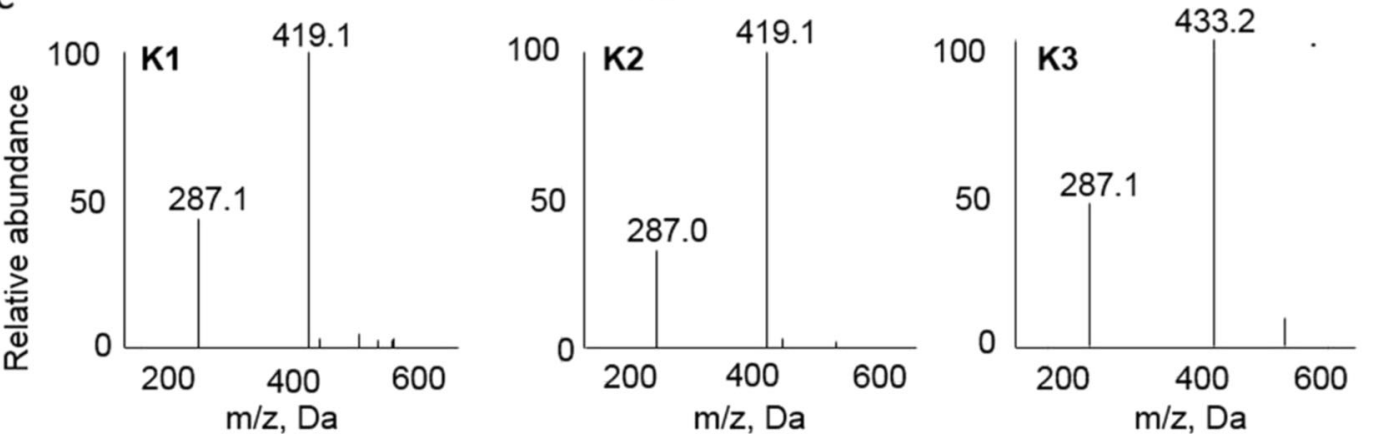

Fig. 2 The chromatogram of flavonoids in petals of Malus and the identification of kaempferol glycosides. a The HPLC chromatogram of flavonoids detected at $365 \mathrm{~nm}$ (M. baccata, white flower genotype; M. 'Royalty', red flower genotype; WT, M. domestica 'Royal Gala'; A1/3, the MYB10 overexpressed transgenic line of 'Royal Gala'). b Structures of kaempferol glycosides. c ESI-MS spectra of three kaempferol glycosides at positive mode. C1: cyanidin 3-O-galactoside; Q1: quercetin 3-O-galactoside; Q2: quercetin 3-O-glucoside; Q3: quercetin 3-O-xyloside; Q4: quercetin 3-Oarabinoside; Q5: quercetin 3-O-rhamnoside; K1, kaempferol 3-O-xyloside; K2, kaempferol 3-O-arabinoside, and K3, kaempferol 3-O-rhamnoside

(Table S1), K1, K2 and K3 were identified as kaempferol 3-O- $\beta$-xylopyranoside (kaempferol 3-O-xyloside), kaempferol 3-O- $\alpha$-arabinofuranoside (kaempferol 3-O-arabinoside) and kaempferol 3-O- $\alpha$-rhamnopyranoside (kaempferol 3-O-rhamnoside), respectively.

\section{Correlation between cyanidin 3-O-glycosides and} kaempferol 3-O-glycosides synthesis in Malus

To investigate whether there is any relationship between anthocyanin and kaempferol glycosides, flavonoids compounds in different tissues of WF and RF were quantified. 

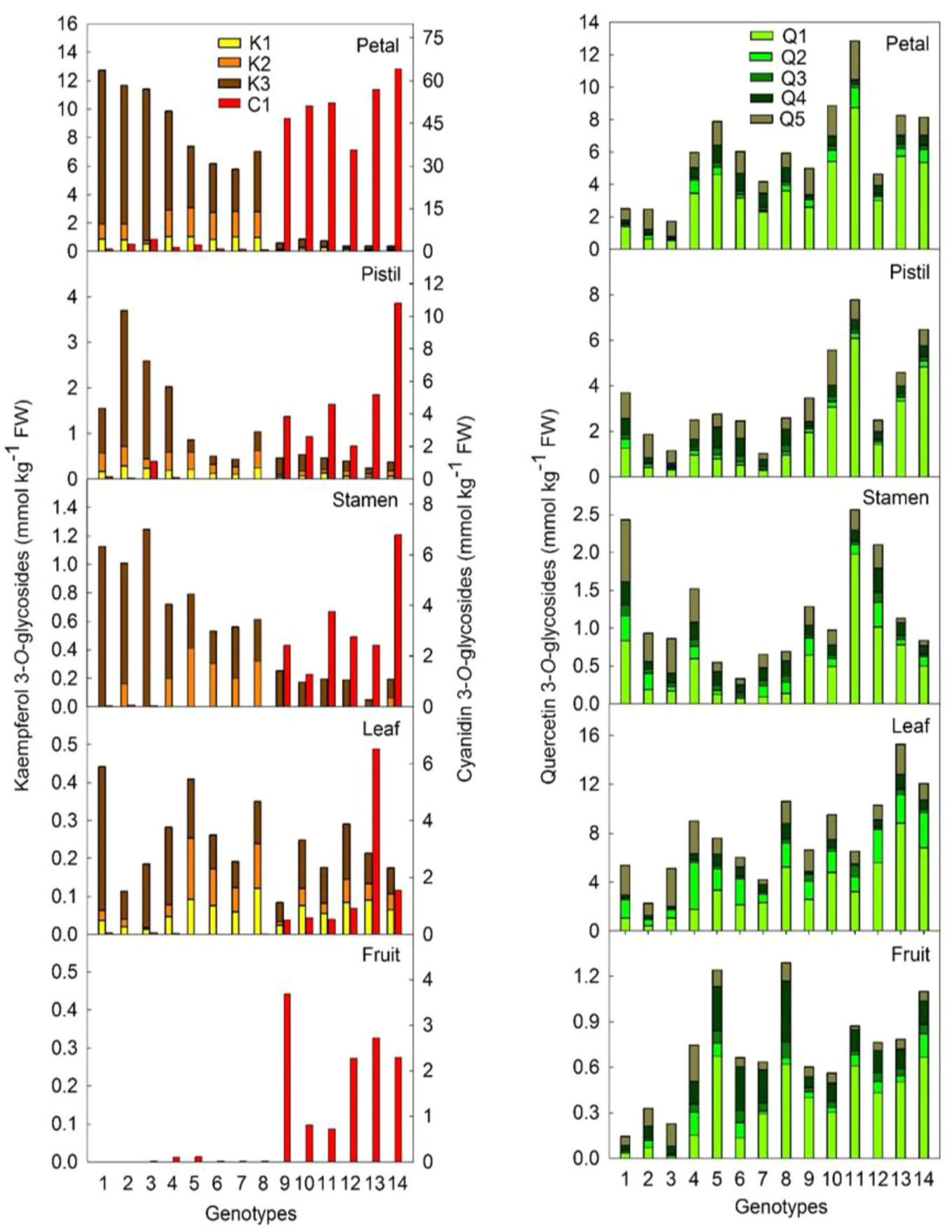

Fig. 3 Flavonoid compounds concentration in different tissues of Malus germplasm resources. 1, M. hupehensis; 2, M. micromalus; 3, M. halliana; 4, M. baccata; 5, M. domestica 'Golden Delicious'; 6, M. domestica 'Fuji'; 7, M. domestica 'Red Delicious'; 8, M. domestica 'Gala'; and red flower genotypes: 9, M. 'Sparkler'; 10, M. 'Radiant'; 11, M. 'Adams'; 12, M. 'Kelsey'; 13, M. 'Perfect Purple'; 14, M. 'Royalty'. C1: cyanidin 3-O-galactoside; K1, kaempferol 3-O-xyloside; K2, kaempferol 3-O-arabinoside; K3, kaempferol 3-O-rhamnoside; Q1: quercetin 3-O-galactoside; Q2: quercetin 3-O-glucoside; Q3: quercetin 3-O-xyloside; Q4: quercetin 3-O-arabinoside; and Q5: quercetin 3-O-rhamnoside. The flower petals were collected at stage 1 as described in the materials and methods. Data are presented as the average of five replicates

The quantitative analysis of kaempferol 3-O-glycosides showed that kaempferol 3-O-rhamnoside was the major kaempferol 3-O-glycosides compound in Malus (Fig. 3). Moreover, kaempferol 3-O-glycosides mainly accumulated in flowers and very little in leaves and fruits. It was also found that kaempferol 3-O-glycosides did not accumulate in pollen, although they accumulated in pollen sacs (Fig. S2). Further analysis showed that the concentrations of 
kaempferol 3-O-glycosides had a significantly negative correlation with that of cyanidin 3-O-glycosides and quercetin 3-O-glycosides (quercetin 3-O-galactoside, quercetin 3-Oglucoside, quercetin 3-O-xyloside, quercetin 3-O-arabinoside, and quercetin 3-O-rhamnoside) in flowers (Fig. S3), but not in leaf and fruit (Fig. 3). Moreover, the concentrations of kaempferol 3-O-glycosides in flowers were significantly higher whereas that of cyanidin 3-O-glycosides and quercetin 3-O-glycosides were significantly lower in WF compared with RF genotypes (Fig. 3, Fig. S4). No significant difference was found in dihydrochalcone (phlorizin, trilobatin, and sieboldin) concentrations between WF and RF genotypes (Fig. S5).

The synthesis of kaempferol competes with that of cyanidin for the same substrates, naringenin or dihydrokaempferol (Fig. S6). To explore the negative correlation between kaempferol 3-O-glycosides and cyanidin 3$O$-glycosides in flowers, the expression of key genes involved in the kaempferol and cyanidin 3-O-glycosides synthesis pathway (Fig. S6), including $M d F 3^{\prime} H I, M d F 3^{\prime}$ HII, MdFLS, MdDFR, and MdANS, were assayed. Among these five genes, $M d F 3^{\prime} H I$ did not show any difference between WF and RF genotypes (Fig. 4, Fig. S7). However, $M d F 3^{\prime} H I I$ and MdFLS showed a significant difference between WF and RF genotypes before full bloom. The expression level of $M d F 3^{\prime} H I I$ was significantly lower, but that of MdFLS was significantly higher in the flowers of WF genotypes compared with that in RF genotypes. No significant difference was found between WF and RF genotypes in the transcript abundance of $M d F 3^{\prime} H I I$ in leaf or fruit, while $M d F L S$ showed higher expression levels in leaves of WF compared with that of RF. Before full bloom, the expression levels of MdDFR and MdANS were higher in the flowers of RF, but similar in leaf and fruit compared with that of WF (Fig. 4, Fig. S7). The MYB10-overexpressed transgenic flowers of 'Royal Gala' showed significant increases in the expression levels of $M d F 3^{\prime} H I I$, but significant decreases in the levels of MdFLS compared with the WT (Fig. 5a, b, Fig. S8a). Moreover, the transgenic flowers accumulated more cyanidin 3-O-galactoside, but less kaempferol 3-O-glycosides (Fig. 5c). Concentrations of dihydrochalcones were comparable in the transgenic and the WT flowers (Fig. S8b).

An RNA interference (RNAi) approach was used to repress the expression of $M d F 3^{\prime} H I I$ in 'GL3' apple seedlings and three transgenic lines were obtained (Fig. 5d). The transformation of $M d F 3^{\prime} H I I$ did not affect the expression level of MdFLS (Fig. S8c). Among the three transgenic lines of 'GL3', two lines showed significantly higher concentrations of kaempferol 3-O-glycosides in the leaves compared with that in the WT. Meanwhile, different from that in the flowers of WF genotypes, the fraction of kaempferol 3-O-rhamnoside to total kaempferol 3-O-glycosides was reduced in the transgenic 'GL3' leaves (Fig. 5f). The concentration of other flavonoids such as quercetin 3-O-glycosides and dihydrochalcones remained unchanged in the transgenic leaves (Fig. 5, Fig. S8d).

\section{Effects of flavonoids on pollen tube growth and seed set}

To investigate whether the flavonoids in pistils affect pollen tube growth in vivo, the MYB10-overexpressed transgenic flowers as well as its WT were pollinated with the pollen grains of 'Granny Smith'. After pollination, it was found that the tube growth of the pollen grains of 'Granny Smith' was significantly slower in the MYB10overexpressed transgenic flowers compared with that in the WT (Fig. 6). At $48 \mathrm{~h}$ after pollination, the pollen tube had arrived at the style region with multicellular trichomes in the WT, but this was not the case in the transgenic flowers. At $72 \mathrm{~h}$, the pollen tube had passed through the end of the style in the WT, but not in the transgenic flowers.

As there was no difference in pollen viability (the ability of pollen to complete post-pollination events and to effect fertilization $^{32}$ ) between WF and RF genotypes (Fig. S9), pollen was treated with different flavonoid compounds in vitro in order to test whether they might affect pollen tube growth (Fig. 7). It was found that quercetin 3-Oglycosides and cyanidin 3-O-galactoside (the major cyanidin 3-O-glycosides in Malus) had little effect on pollen tube growth, while kaempferol and kaempferol 3-O-glycosides, especially kaempferol 3-O-rhamnoside, enhanced the growth four hours after treatment. Moreover, the kaempferol 3-O-rhamnoside treatments in vivo significantly increased the amount of well-developed seed for two RF genotypes, $M$. 'Adams' and $M$. 'Radiant', while cyanidin 3-O-galactoside did not affect the amount of well-developed seed for two WF genotypes, M. micromalus and M. halliana (Fig. 8).

\section{Transcriptomic analysis of treated pollen}

To further investigate the effect of kaempferol 3-Orhamnoside on pollen tube growth, transcriptome analysis was carried out using the pollen (including pollen tubes) treated with and without kaempferol 3-O-rhamnoside for $4 \mathrm{~h}$ in vitro, with pollen at $0 \mathrm{~h}$ being set as control. An intersection was taken among the differentially expressed genes (DEGs) between 4 and $0 \mathrm{~h}$ without kaempferol 3-Orhamnoside treatment (T0_Mock), the DEGs between 4 and $0 \mathrm{~h}$ with kaempferol 3-O-rhamnoside treatment (T1_Mock), and the DEGs between $4 \mathrm{~h}$ with and without kaempferol 3-O-rhamnoside treatment (T1_T0). The DEGs with similar expression trends were selected. Only 41 DEGs $\left(\log _{2}{ }^{x}>0.2\right)$ were found, including 32 upregulated and nine downregulated genes (Fig. 9, Table S2).

Among the DEGs, there were six transcription factors (TF), including two WD40 (MD03G1216600, 


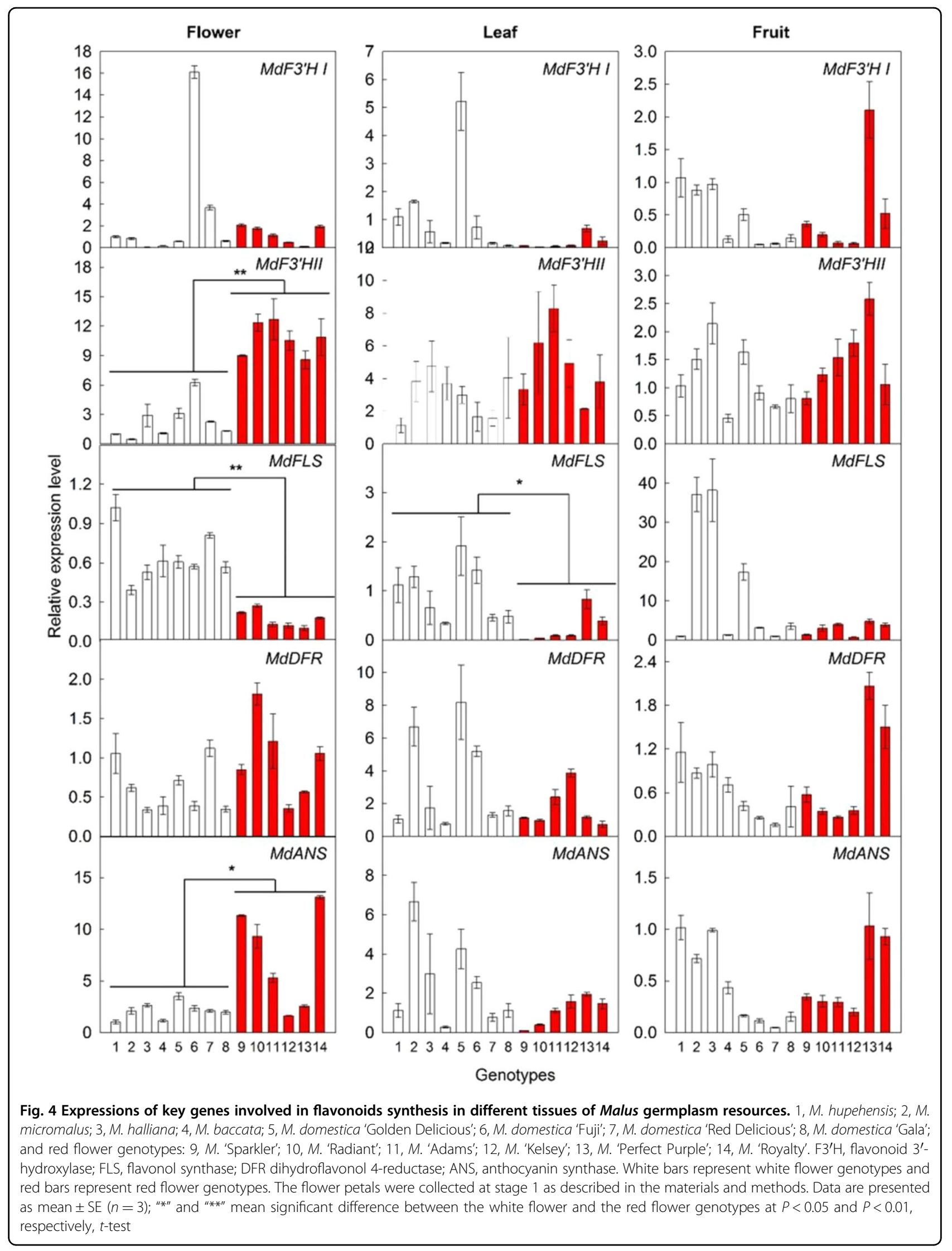




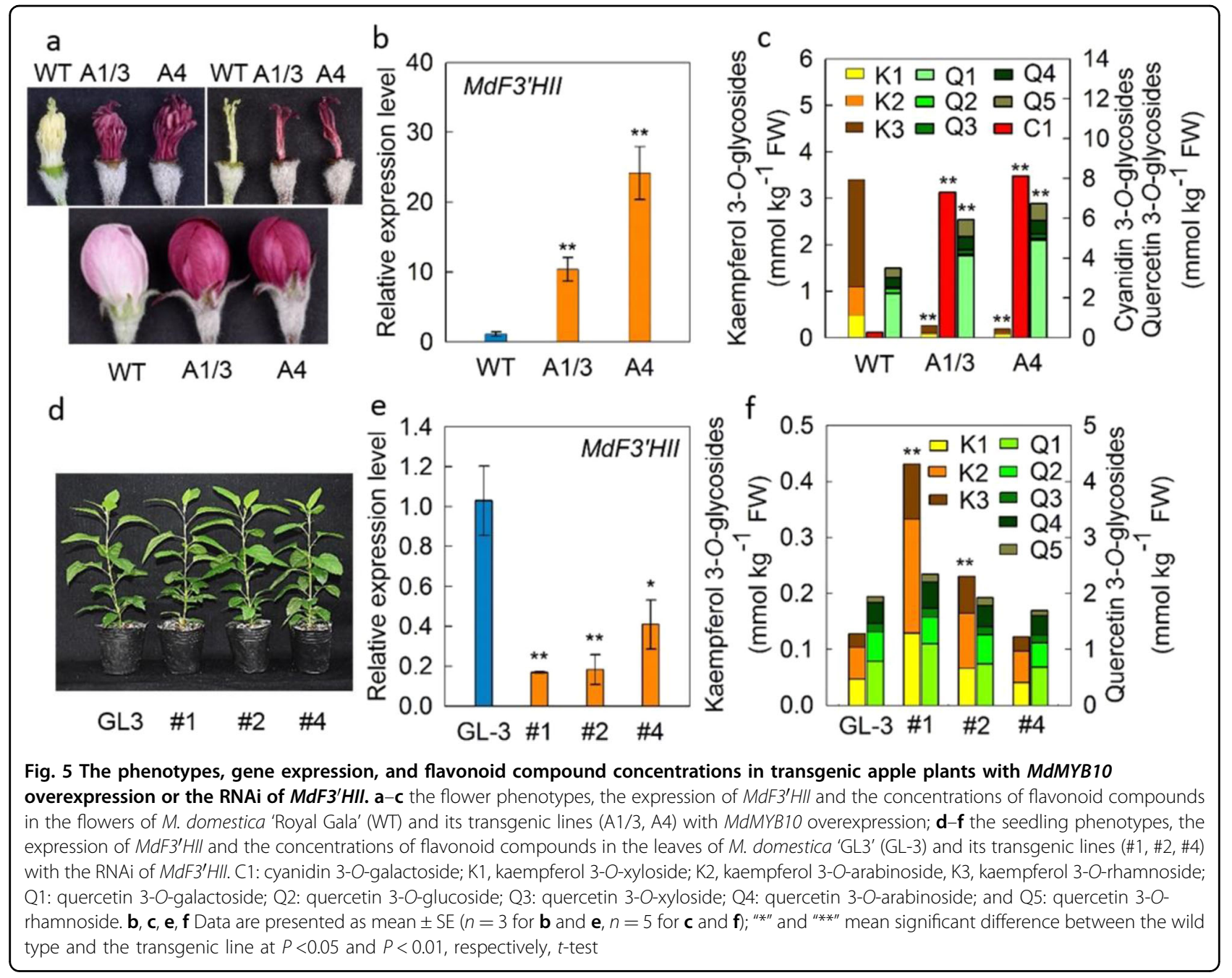

MD00G1078300), one NAC (MD09G1123300), one TF with BTB/POZ domain (MD11G1039500), and two TFs with histone-fold (MD03G1273800, MD02G1134400). The DEGs also included four low-molecular weight heat shock proteins (HSPs) (MD01G1144400, MD07G1210800, MD15G1053800, MD08G1068000); four high molecular weight HSPs (MD17G1226000, MD17G1226100, MD01G1126500, MD01G1208700); one activator of HSP 90 ATPase (MD12G1055300); one AAA-type ATPase (MD06G1201600); six genes involved in small GTPase and MAPK mediated signal transduction: one rab escort protein (MD10G1065000), one guanine nucleotide exchange factor (MD05G1233200), two serine/threonine protein kinase (MD00G1151100, MD04G1187300), one phosphatidylinositol transfer protein (MD05G1062600), and one phosphatidylinositol-mediated signaling protein (MD04G1176700). There were four genes involved in microtubule-based movement: two kinesin proteins (MD06G1067900, MD08G1078700) and two microtubule- associated movement proteins (MD14G1216600, MD03G1268600); and five mitochondrial genes: two pentatricopeptide repeat superfamily proteins (MD13G1197300, MD15G1002100), one prohibition (MD09G1016800), one mitochondrial inner membrane protein (MD15G1329400), and one synaptotagmin (MD00G1161000). Other DEGs included two ubiquitin genes (MD08G1047300, MD13G1214800); three genes involved in carbohydrate metabolism (MD10G1182800, MD17G1157900, MD13G1148300); three genes involved in glycolipid and anions transport (MD00G1076100, MD16G1221400, MD12G1100900); one gene involved in sterol biosynthesis (MD02G1190300); and one uncharacterized gene (MD05G1259800). The expression of some genes, especially HSPs and two genes related to small GTPase, were doubly checked using real-time qPCR (Fig. S10). The qPCR results showed that most of the differences in the gene expressions were consistent with that obtained from transcriptome analysis. 

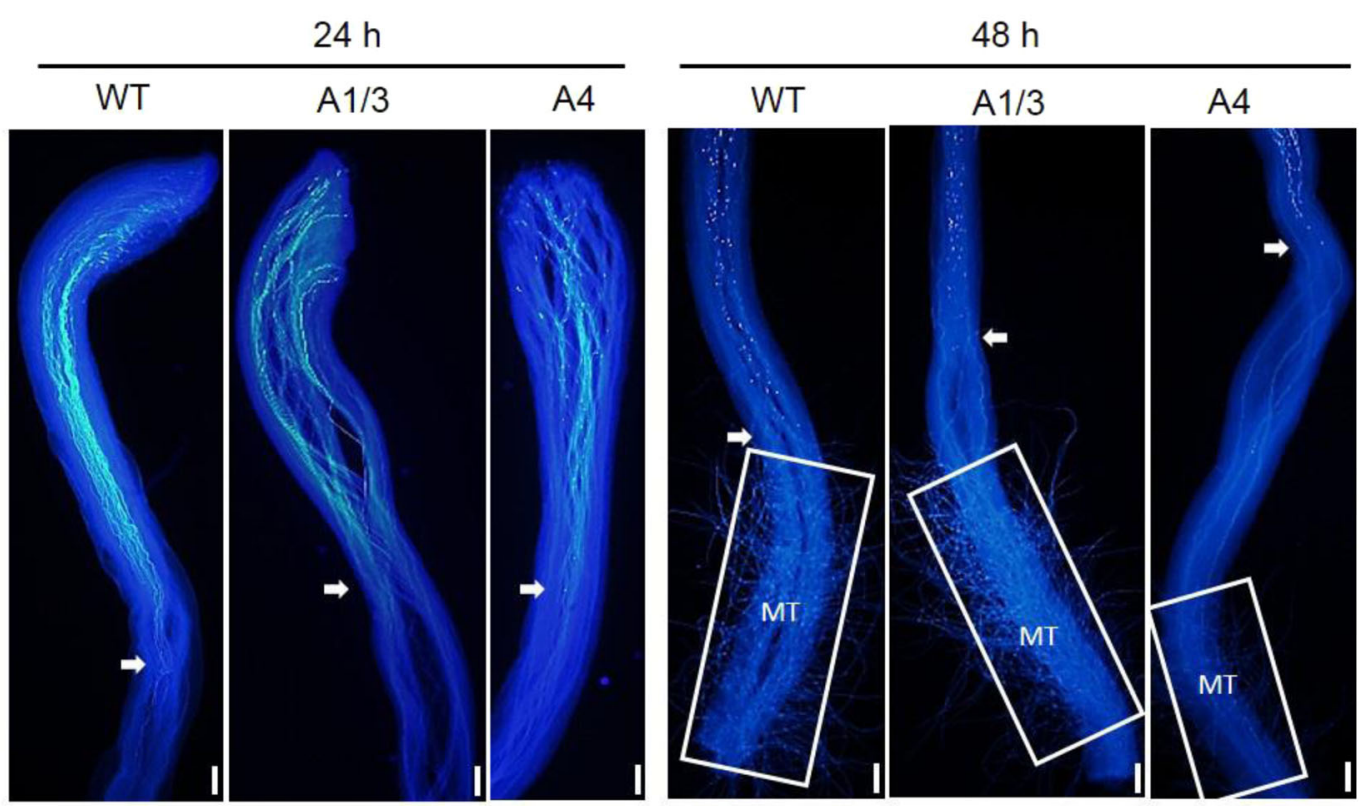

$72 \mathrm{~h}$
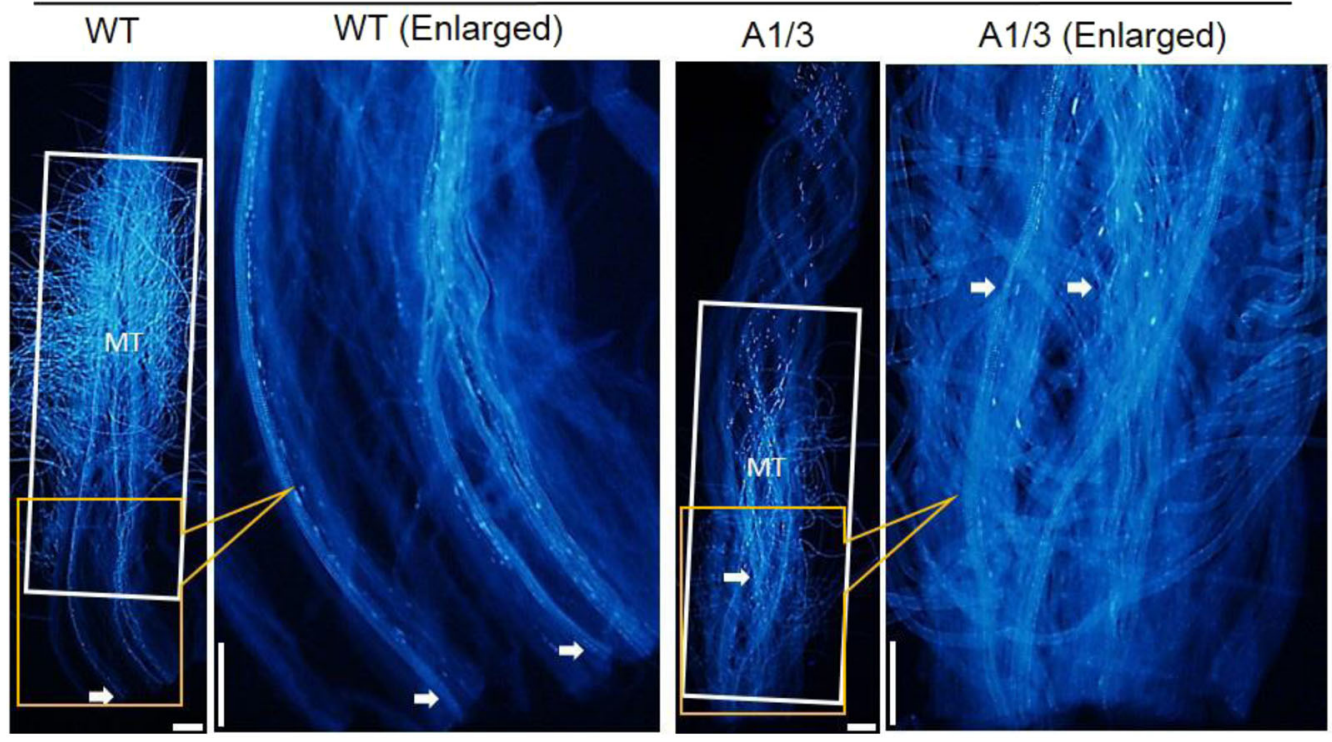

Fig. 6 The tube growth of Malus domestica 'Granny Smith' pollen in flowers of M. domestica 'Royal Gala' and its transgenic lines with MdMYB10 overexpression. The stained pollen tubes were observed with a fluorescence microscope at 24, 48, and $72 \mathrm{~h}$ after pollination. WT, wild type; $A 1 / 3$ and $A 4$, transgenic lines. The white arrows indicate the locations where pollen tubes arrived; $M T$ : multicellular trichome, Bars $=50 \mu m$

\section{Effects of auxin and calcium on pollen tube growth}

The application of $\beta$-naphthoxyacetic acid (NOA), an inhibitor of auxin influx from plant cells; $1-N$-naphthylphthalamic acid (NPA), an inhibitor of auxin efflux from plant cells; and EGTA, a chelator of calcium ion, all significantly inhibited pollen tube growth (Fig. 10a). These three chemical treatments also lowered the abundance of calcium ions at the tip of pollen tubes
(Fig. 10b). The enhancement of pollen tube growth and calcium ion abundance by kaempferol 3-O-rhamnoside treatment also disappeared with the combined NOA, NPA, or EGTA treatment. It was also found that the NOA and NPA treatments lowered the expression levels of genes encoding HSPs and those involved in the small GTPase signaling pathway, while EGTA either did not change or increased their expression (Fig. 10c). 


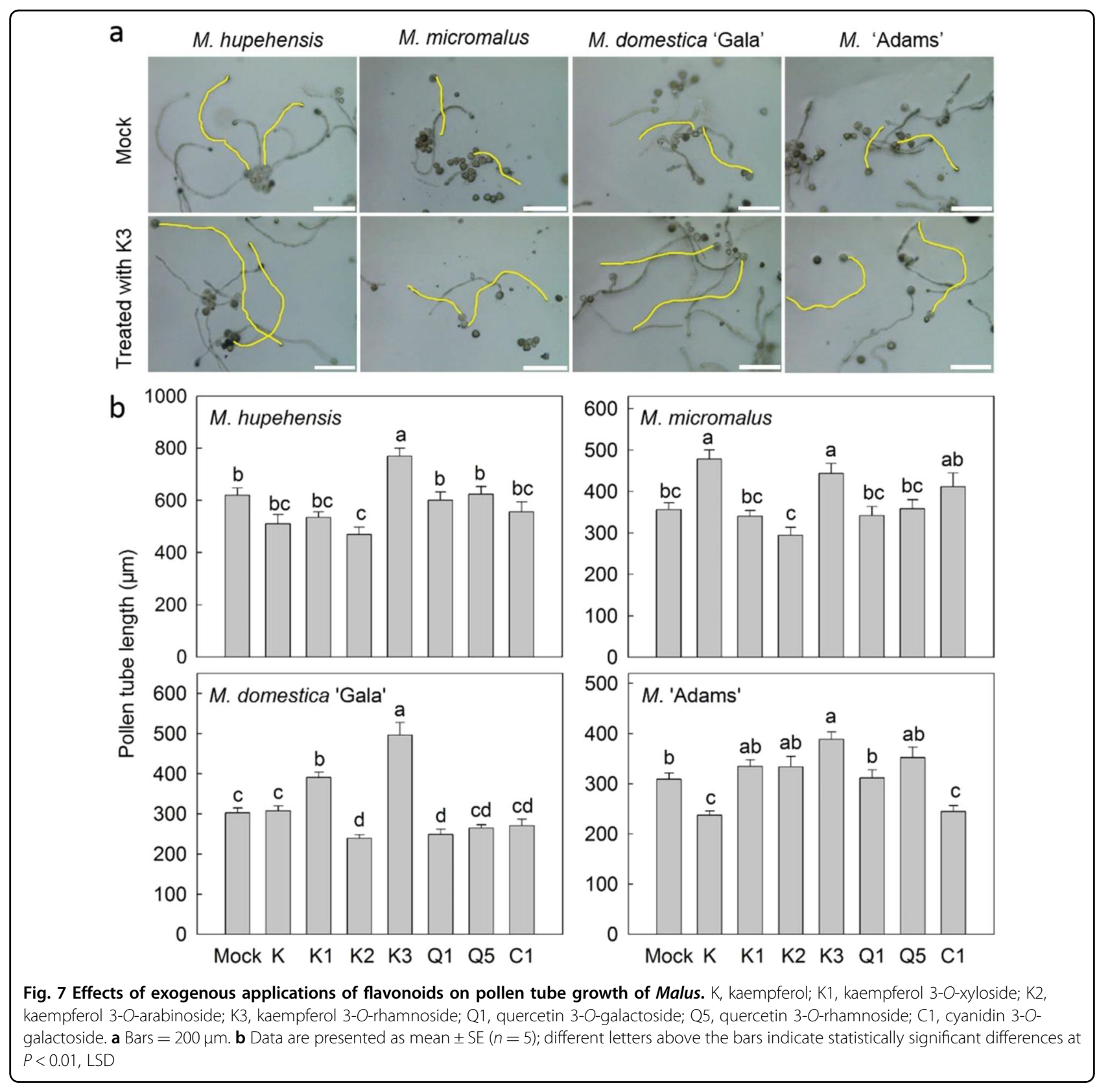

\section{Discussion}

Our present study demonstrated that the biosynthesis of kaempferol 3-O-glycosides competed with that of anthocyanin in the flowers of Malus, which partly determined the flower color (Figs. 1-5). Moreover, kaempferol 3-Oglycosides, especially kaempferol 3-O-rhamnoside, was involved in the regulation of pollen tube growth and seed set (Figs. 6-10).

Dihydrochalcones, cyandin 3-O-galactoside, kaempferol 3-O-glycosides, and quercetin 3-O-glycosides were the major flavonoid compounds detected in the flowers of Malus. As the concentration of dihydrochalcones was comparable between WF and RF genotypes (Fig. S5), it is unlikely that $C H S$ and the upstream structural genes in the pathway may play a role in the different kaempferol 3-Oglycosides and anthocyanin concentrations between WF and RF genotypes. Compared with quercetin, kaempferol lacks one more hydroxyl group at the $3^{\prime}$-position of the Bring, so the glycosylation at the 3-position of the C-ring of kaempferol and quercetin might be catalyzed by the same UGT gene. At different developmental stages, the concentrations of quercetin 3-O-glycosides were comparable or even lower in the flowers of WF compared with that of RF genotypes (Fig. 3, Fig. S4). So, the difference in the 


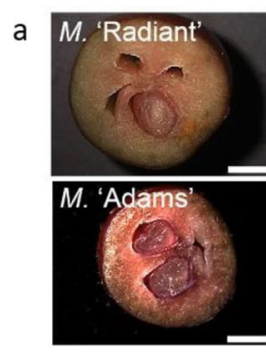

Mock

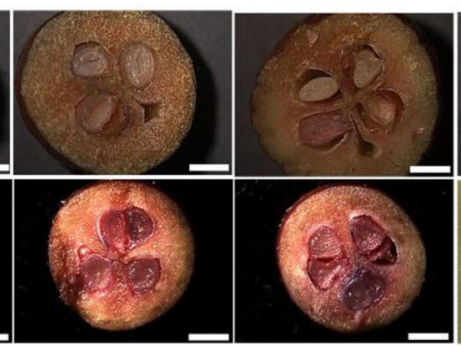

$50 \mu \mathrm{M} \mathrm{K} 3$
$100 \mu \mathrm{M} \mathrm{K3}$

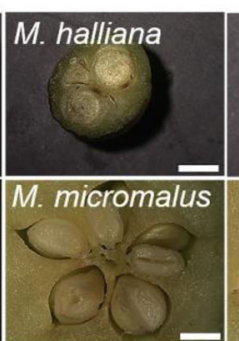

Mock

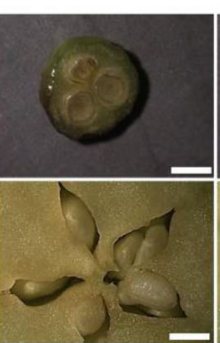

$50 \mu \mathrm{M} \mathrm{C1}$

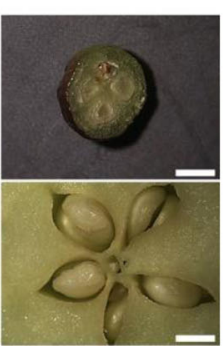

$100 \mu \mathrm{M} \mathrm{C1}$

b
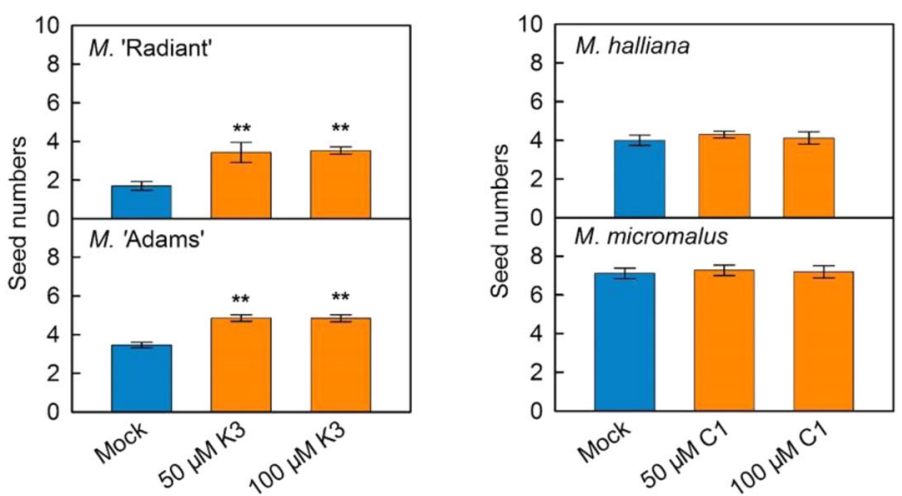

Fig. 8 Effect of exogenous applications of flavonoids on seed set of Malus. Red flower genotypes, M. 'Radiant' and M. 'Adams'; white flower genotypes, $M$. halliana and $M$. micromalus. K3, kaempferol 3-O-rhamnoside; C1, cyanidin 3-O-galactoside. a Bars $=2 \mathrm{~mm}$. $\mathbf{b}$ Data are presented as mean $\pm \mathrm{SE}(n=5)$; "**" above the bars indicate statistically significant differences between mock and treatment at $P<0.01, t$-test

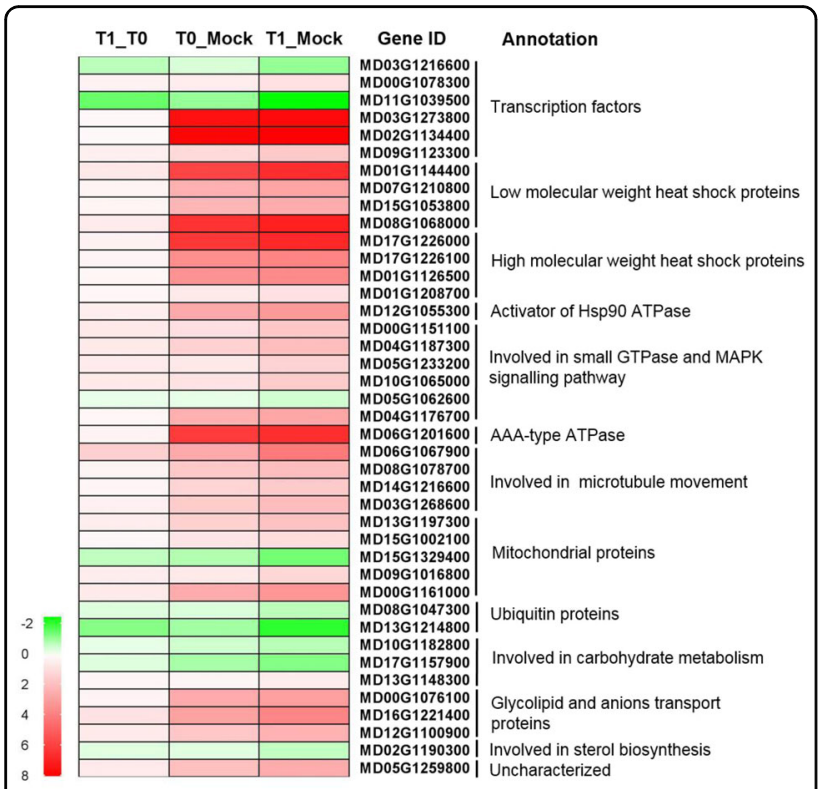

Fig. 9 Gene expressions during pollen tube growth after different treatments. Mock: pollen cultured for $0 \mathrm{~h}$, T0: pollen cultured for $4 \mathrm{~h}$, T1: pollen cultured for $4 \mathrm{~h}$ with $1 \mu \mathrm{M}$ kaempferol 3-Orhamnoside treatment concentrations of kaempferol 3-O-glycosides between WF and RF should not necessarily relate to glycosylation.

In previous studies, it was found that the overexpression of both $M d F 3^{\prime} H I$ and $M d F 3^{\prime} H I I$ in either the Arabidopsis $t t 7$ mutant or tobacco would recover the seed pigmentation of the $t t 7$ mutant or result in more anthocyanin accumulation in tobacco flowers ${ }^{3}$. Tian et al. also reported that the everred leaf colouration of crabapple was regulated by $M Y B 10$ TF through regulating the expression level of $F 3^{\prime} H^{33}$. However, based on the gene expression levels in the different Malus genotypes as well as that in the MYB10-overexpressed 'Royal Gala' transgenic lines, the competition between anthocyanin and kaempferol 3-O-glycosides synthesis in Malus flowers might be controlled by the genes such as F3'HII, FLS, DFR, and ANS (Fig. 4, Fig. S7). For anthocyanin and kaempferol 3-O-glycosides synthesis, $F 3^{\prime} H$ and $F L S$ are the key enzymes controlling the metabolic flux using the shared substrates such as naringenin or dihydrokaempferol (Fig. S6). So, compared with DFR and ANS, $F 3^{\prime} H I I$ and $F L S$ might contribute more to the competition. $M d F 3^{\prime} H I$ has $91 \%$ nucleotide sequence identity in the coding region to $M d F 3^{\prime} H I I$ (Fig. S11), but unlike $F 3^{\prime} H I I$, the expression of $F 3^{\prime} H I$ in flowers was not significantly different between WF and RF genotypes (Fig. 4). The overexpression of $M Y B 10$ hardly changes the expression of $F 3^{\prime} H I$ compared 


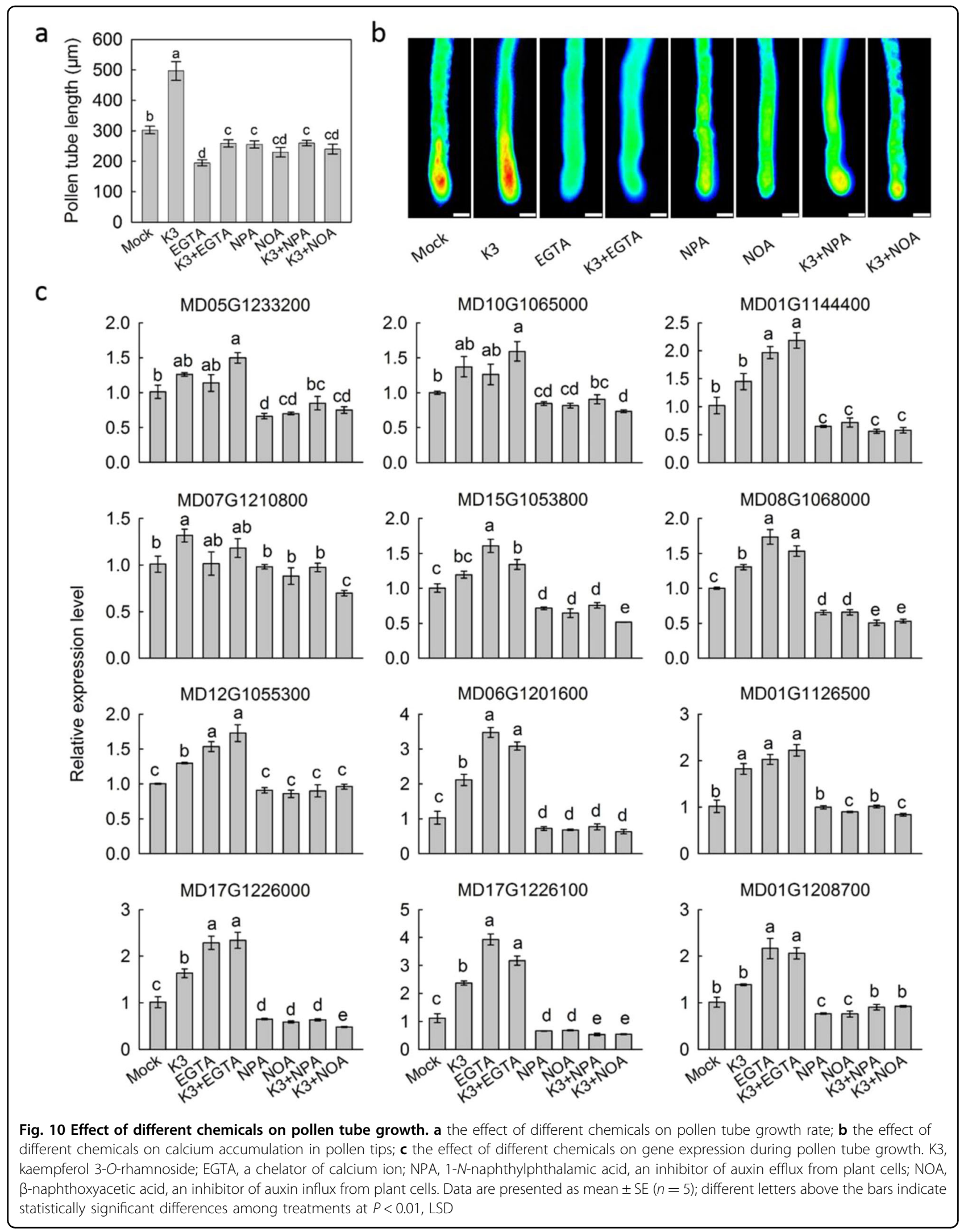




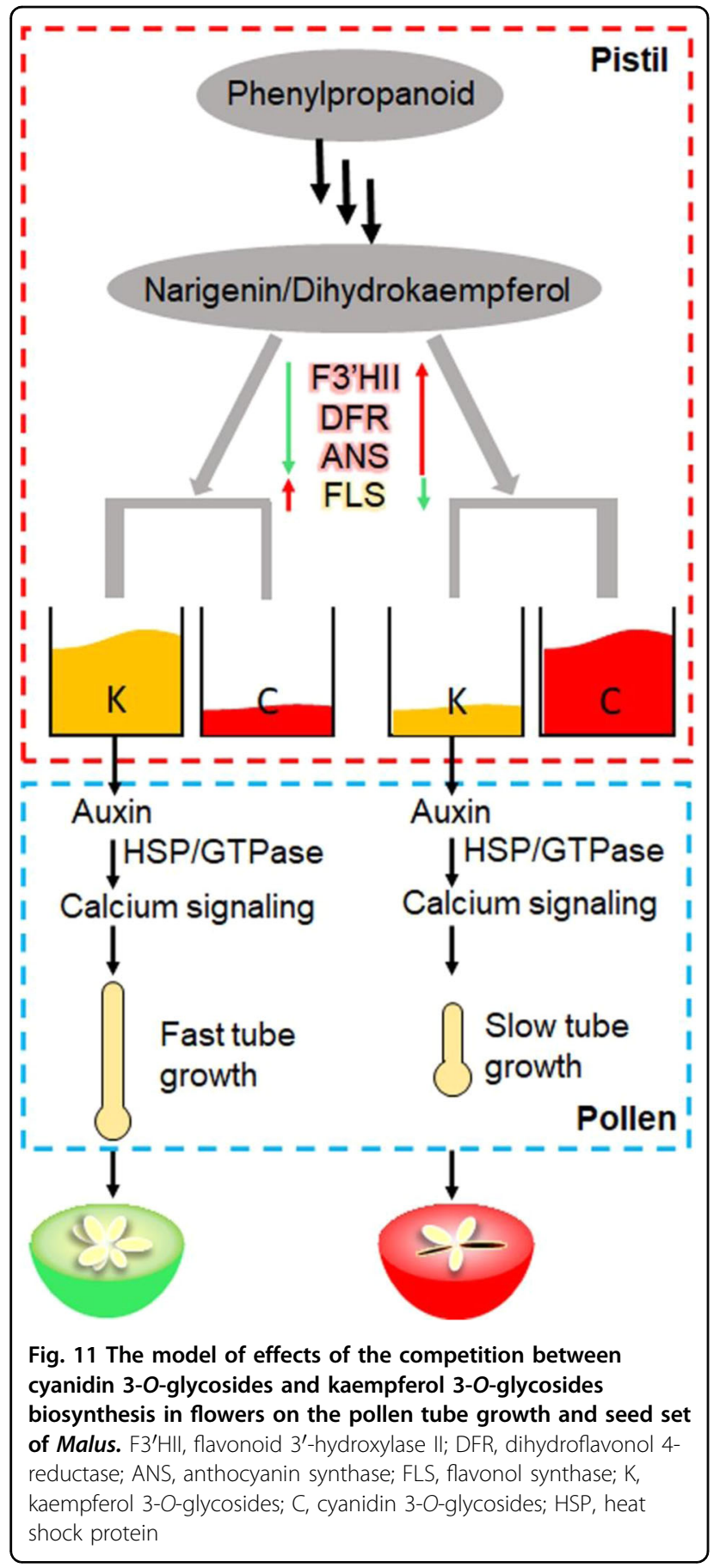

with that of $F 3^{\prime} H I I$ in the red flowers of 'Royal Gala' either (Fig. S8).

Interestingly, it was found that kaempferol 3-O-glycosides accumulated mainly in flowers but not in leaves or fruits of Malus (Fig. 3). The function of compounds usually relates to the tissues/organs they are accumulated in. In the leaves, whilst expression of $F 3^{\prime} H I I$ was downregulated sharply, the level of kaempferol 3-O-glycosides only increased a small amount (Fig. 5d-f). This suggests kaempferol 3-O-glycosides should be functional mainly in flowers. This might also explain why the concentrations of quercetin 3-O-glycosides remained unchanged in the transgenic leaves (Fig. 5f). Recently, it was reported that flavonols in the pollen of tomato might control pollen tube growth and integrity by regulating ROS homeostasis during high-temperature stress $^{23}$. Meng et al. reported that decreased sorbitol synthesis might lead to abnormal stamen development and reduced pollen tube growth ${ }^{34}$. In the present study, the difference in kaempferol 3-O-glycosides concentration between WF and RF did not affect pollen viability (Fig. S9). Moreover, no kaempferol 3-O-glycosides was found in the pollen of Malus (Fig. S2). This suggests that before pollination, the development of pollen was not affected by kaempferol 3-O-glycosides or sorbitol in the coat of pollen sacs. After pollination, the reduced kaempferol 3-O-glycosides concentration associated with MYB10-overexpression in 'Royal Gala' appeared to slow pollen tube growth (Fig. 6), demonstrating the regulation of pollen tube growth by kaempferol 3-O-glycosides in pistils. The effects of various flavonoid compounds on pollen tube growth in vitro also clearly showed that it was kaempferol 3-O-glycosides, especially kaempferol 3-O-rhamnoside rather than cyanidin 3-O-glycosides or quercetin 3-O-glycosides, that regulated pollen tube growth of Malus (Fig. 7).

Pollen tubes interact extensively with pistils during growth. Efficient tip growth requires structural organization and vesicular trafficking activities to be highly coordinated in pollen tubes, implying a requirement for signaling networks to coordinate these subcellular behaviors during pollen-pistil interactions. For instance, the signaling pathways involving ROP GTPases, calcium, and phosphoinositides have been demonstrated to coordinate pollen tube growth by regulating cellular activities, such as actin dynamics, exocytosis, and endocytosis ${ }^{35}$. Recently, it was found that the pollen tube tip-localized Pollen receptor-like kinase 6 (PRK6) regulated the direction of pollen tube tip growth as an essential receptor for AtLURE1 signaling ${ }^{36}$, while the ADAPTOR PROTEIN-3 (AP-3) complex-mediated pollen tube growth in Arabidopsis by coordinating vacuolar targeting and organization $^{37}$. In the present study, it was found that kaempferol 3-O-rhamnoside might regulate the pollen tube growth through the ROP GTPases, calcium and phosphoinositide signaling pathway, supported by gene expression data and calcium imaging results (Figs. 9, 10). Moreover, the present study also indicates that kaempferol 3-O-rhamnoside regulated these signaling pathways through modifying auxin transport (Fig. 10).

Auxin has been shown to be critical for pollen development. External applications of auxin can stimulate in vitro pollen tube growth of Nicotiana tabacum and Torenia fournieri $^{38,39}$. Studies on the pollen-specific auxin efflux carrier PIN8 also support a role for auxin in pollen tube 
growth $^{40,41}$. HSPs were suggested to be involved in the thermal tolerance of pollen tubes at high temperatures ${ }^{42}$. Interestingly, in our studies, it was observed that some HSPs were involved in the auxin signaling pathway during the regulation of pollen tube growth (Figs. 8-10). With the inhibition of auxin transport, the expression levels of HSPs were downregulated, while the levels were either unchanged or enhanced when calcium signaling was blocked. These results suggest that HSPs were downstream of auxin signaling but upstream of the calcium signaling pathway. It has been reported that there are over 100 receptor-like kinases expressed in pollen tubes, most of which are expected to be the receptors of small peptides ${ }^{43}$. Functioning as lowmolecular-weight signaling peptides, HSPs-receptor signaling modules appear to be predominant in pollen tube growth. We would not expect reactive oxygen species signaling to be involved in the regulation of pollen tube growth, as kaempferol 3-O-glycosides has only one hydroxyl group while quercetin 3-O-glycosides and cyanidin 3-O-galactoside have an $o$-dihydroxyl at the B-ring. The $o$-dihydroxyl at the B-ring means relatively higher antioxidant capacity ${ }^{25,26}$, and the determinacy to react with the reactive oxygen species.

Pollen tube growth is the main factor which influences seed set ${ }^{44,45}$. Flavonols could regulate seed set in tomato through affecting pollen tube growth ${ }^{23}$. So, it is possible that the exogenous application of kaempferol 3-O-rhamnoside could increase the amount of well-developed seeds in the RF genotypes of Malus (Fig. 8), and account for transgenic 'Royal Gala' red fruit having less welldeveloped seeds (Fig. 1). For RF genotypes, less kaempferol 3-O-glycosides resulted in less well-developed seeds, which would mean lower reproductive capacity in natural conditions. Other studies report that bees seem to be the most important pollinator for apple, and prefer yellow or white flowers with UV absorption properties ${ }^{46-49}$. Therefore, the WF genotypes might also have an advantage in attracting bees for pollination compared with the RF genotypes of Malus. Taken together, this may go some way to explaining why surviving wild Malus species have predominantly white flowers.

In summary, we showed that $F 3^{\prime} H I I, F L S, D F R$, and ANS might control the competition between kaempferol glycosides and anthocyanin biosynthesis in Malus flowers (Fig. 11). Kaempferol glycosides presence in pistils coordinated auxin transport, the gene expression of HSPs and ROP GTPases, and calcium signaling in pollen tubes, affecting pollen tube growth and seed set of Malus.

\section{Materials and methods}

\section{Plant materials and isolation of compounds}

Fourteen Malus genotypes including four apple cultivars ( $M$. domestica 'Golden Delicious', $M$. domestica 'Fuji', $M$. domestica 'Red Delicious', $M$. domestica 'Gala'), four wild species (M. hupehensis, M. micromalus, M. halliana,
M. baccata), and six ornamental crabapple cultivars $(M$. 'Sparkler', $M$. 'Radiant', $M$. 'Adams', $M$. 'Kelsey', $M$. 'Perfect Purple', $M$. 'Royalty') were used in this study. They were grown in the horticultural experimental orchard of Northwest A\&F University, Yangling, Shaanxi, China. All trees were grown under standard horticultural practices with disease and pest control. Flower petals were collected at different developmental stages (stage 1, 6 days before full bloom; stage 2, 3 days before full bloom; and stage 3, full bloom). At stage 3, the four apple cultivars and four wild species had white flowers while at stages 1 and 2, the flowers were pink. The six ornamental crabapple cultivars always had red flowers. Pistils, stamens, and pollens were collected 1 day before full bloom. New fully expanded leaves were collected at the full bloom stage, while fruit were collected at $\sim 40$ days after full bloom. Five replicates (one tree for each replicate. For each tree, 20 flowers, fruits, or leaves were taken from the west part of tree canopies at each sampling time) of all tissues were collected and immediately frozen in liquid nitrogen, then ground to powder and mixed in liquid nitrogen with an $\mathrm{Al}$ 1 grinder from IKA ${ }^{\circledR}$ works (VWR, Radnor, PA, USA), and stored at $-80^{\circ} \mathrm{C}$ until use. Pollen were dried at $28^{\circ} \mathrm{C}$ for $24 \mathrm{~h}$ and stored at $-20^{\circ} \mathrm{C}$ until use. The $M d M Y B 10$ overexpressed 'Royal Gala' apple lines and the corresponding WT trees were grown in glasshouse conditions, as described by Espley et al. ${ }^{31}$.

For compound isolation, $5 \mathrm{~kg}$ of $M$. domestica 'Fuji' flowers and $0.8 \mathrm{~kg}$ of $M$. 'Perfect Purple' leaves were collected. The dried flowers were used to isolate kaempferol derivative compounds and quercetin derivative compounds (quercetin 3-O-galactoside and quercetin 3-Orhamnoside) whereas the leaves were used for cyanidin 3$\mathrm{O}$-galactoside isolation, as described by Xiao et al. ${ }^{25}$ with minor modifications. The isolated kaempferol derivative compounds (purity > 98\%) were identified using LC-ESIMS and NMR as described by Xiao et al. ${ }^{25}$.

\section{Transformation of apple}

A line with high regeneration capacity isolated from 'Royal Gala' (M. domestica) named 'GL-3'50 was used for genetic transformation with $M d F 3^{\prime} H I I a$. Transgenic 'GL3' apple plants were generated from leaf fragments through Agrobacterium-mediated transformation, as previously described ${ }^{50}$. The vector pK7GWIWG2 driven by the $35 \mathrm{~S}$ promoter was used as the RNA interference vector for $M d F 3^{\prime} H I I a$. The $M d M Y B 10$ overexpressed 'Royal Gala' apple plants and the corresponding WT were as described by Espley et al. ${ }^{31}$.

\section{Flavonoid compound analysis}

The extraction and analysis of flavonoid compounds in Malus were carried out as described by $\mathrm{Li}$ et al. ${ }^{51}$. The isolated compounds with the purity over $98 \%$ with 
different concentrations were used to make standard curves to calculate the concentrations of each compound in plants.

\section{Seed set analysis}

Fruits of Malus were harvested 60 days after full bloom, with 60 fruits from 5 trees being randomly selected, seeds extracted and a knife cut to the side of the seed used to reveal the ventricle. For the transgenic fruit and its wild type, 15 fruit were, respectively, selected randomly for seed set analysis. The image of seed sets was observed using a stereoscopic microscope (Leica MZ10F, Germany).

\section{Flavonoid compounds treatment and phenotypic analysis of pollen tube growth in vitro}

Harvested pollen grains were cultured on solid germination medium (GM, $10 \%$ sucrose, $0.01 \%$ boric acid, $1 \%$ agar, $\mathrm{pH}$ 6.5) as described by Meng et al. with minor modification $^{34}$. For compound treatment, $1 \mu \mathrm{M}$ kaempferol, kaempferol derivatives, quercetin derivatives, or cyanidin 3-O-galactoside dissolved in Dimethylsulfoxide (DMSO) were added into the GM and mixed uniformly before the media solidified. The DMSO solution without additives was used as the control. The pollen was incubated at $28^{\circ} \mathrm{C}$ in a dark and humid environment for $4 \mathrm{~h}$.

The pollen of $M$. domestica 'Fuji' was used for $1-N$ naphthylphthalamic acid (NPA, final concentration, $40 \mu \mathrm{M}$ ), $\beta$-naphthoxyacetic acid (NOA, final concentration $20 \mu \mathrm{M}$ ), and EGTA (final concentration $10 \mathrm{mM}$ ) treatments. In our preliminary experiment, it was found that EGTA fully inhibited the germination of pollen, so the EGTA treatments were carried out after $2 \mathrm{~h}$ of culture of pollen.

The calcium fluorescence and pollen tube length were observed with a fluorescence microscope (BX51 + DP70, Olympus). The length of pollen tube was measured by ImageJ. Measurement of the length of pollen tubes was carried out on five replicates. For each replicate, 50 pollen grains were randomly selected.

\section{Phenotypic analysis of pollen tube growth in vivo}

Pollen grains from $M$. domestica 'Granny Smith' were collected to pollinate 'Royal Gala' and its MYB10 overexpressed transgenic flowers. The pistils were harvested 24,48 , and $72 \mathrm{~h}$ after pollination, and then fixed in FAA fixative solution (70\% alcohol: acetic acid: formaldehyde $=18: 1: 1)$ for $12 \mathrm{~h}$. The fixed pistils were washed with distilled water three times following an incubation in $2 \mathrm{M}$ $\mathrm{NaOH}$ softening solution for $24 \mathrm{~h}$. After being washed with distilled water three times, the pistils were stained in $0.1 \%$ aniline blue $\left(0.1 \% \mathrm{~K}_{3} \mathrm{PO}_{4}\right.$ buffer $)$ for $24 \mathrm{~h}$ in the dark. Pollen tubes in the style were visualized with a BX51+ DP70 fluorescence microscope (Olympus Corporation,
Japan) as described by Kho and Baer $^{53}$ with minor modifications.

\section{RNA extraction, qRT-PCR, and RNA-seq analyses}

Total RNA was isolated using the SDS-phenol method according to Malnoy et al. $^{52}$. All qRT-PCR experiments were performed with the Bio-Rad CFX96 system (Bio-Rad Laboratories, Hercules, CA, USA). The primers for

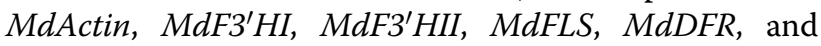
MdANS were shown in Supplemental Table S3. The total RNA was also submitted to Novogene in Beijing (https:// en.novogene.com/) for library construction and RNA sequencing. Unigenes with adjusted $P$-value $<0.05$ and fold change $\log _{2}{ }^{x}>0.2$ were identified as DEGs. Three biological replicates were performed for the analysis.

\section{Chemical feeding to stigma in vivo}

One day before full bloom, 50 or $100 \mu \mathrm{M}$ kaempferol 3$O$-rhamnoside was dropped onto the stigma of two red flower genotypes, $M$. 'Adams' and $M$. 'Radiant', while 50 or $100 \mu \mathrm{M}$ cyanidin 3-O-galactoside was dropped to the stigma of two white flower genotypes, $M$. micromalus and $M$. halliana. The flowers treated with the DMSO solution without additives were used as a control. Forty days after feeding treatment, the seed set was observed and counted. For each treatment, 60 flowers from five trees were randomly selected for feeding treatment. The fruit setting rate was $100 \%$.

\section{Statistical analysis}

All data were analyzed with $t$-test or least significant difference (LSD) using SPSS 16.0 software (SPSS, Chicago, IL, USA).

\section{Acknowledgements}

This work was funded by the National Key R\&D Program (2018YFD1000200) and the National Natural Science Foundation (31972366) of China. We thank the technicians at the State Key Laboratory of Crop Stress Biology for Arid Areas, Northwest A\&F University, Yangling, for help running the NMR (Hongli Zhang), LC-MS (Liru Jian), microscopic imaging (Guoliang Pei and Hua Zhao), and gPCR (Xueling Huang) analyses. We also thank Caitlin Elborough at Plant \& Food Research for help calculating the seeds quantities of the transgenic fruit.

\section{Author details \\ 'State Key Laboratory of Crop Stress Biology for Arid Areas/Shaanxi Key Laboratory of Apple, College of Horticulture, Northwest A\&F University, Yangling, Shaanxi 712100, China. ${ }^{2}$ College of Food Science and Technology, Northwest University, Xi'an, Shaanxi 710069, China. ${ }^{3}$ The New Zealand Institute for Plant and Food Research Ltd, Private Bag, 92169 Auckland, New Zealand}

\section{Author contributions}

W.C., Z.X., F.M., and P.L. designed the research; W.C. did the transcriptomic, HPLC, GL3 transformation, and biochemical analyses; Z.X. and J.W. performed the compounds isolation and identification; Y.W., R.Z., K.L.-W., and R.E. assayed the MYB10 overexpressed apples. W.C. Z.X., and P.L. analyzed data and wrote the paper. K.L.-W. and R.E. revised the paper.

Conflict of interest

The authors declare no competing interests. 
Supplementary information The online version contains supplementary material available at https://doi.org/10.1038/s41438-021-00609-9.

Received: 6 December 2020 Accepted: 20 May 2021

Published online: 01 August 2021

\section{References}

1. Winkel-Shirley, B. Flavonoid biosynthesis. A colorful model for genetics, biochemistry, cell biology, and biotechnology. Plant Physiol. 126, 485-493 (2001)

2. Dare, A. P. et al. Overexpression of chalcone isomerase in apple reduces phloridzin accumulation and increases susceptibility to herbivory by twospotted mites. Plant J. 103, 293-307 (2020).

3. Han, Y. et al. Ectopic expression of apple $F 3^{\prime} H$ genes contributes to anthocyanin accumulation in the Arabidopsis $t 7$ mutant grown under nitrogen stress. Plant Physiol. 153, 806-820 (2010).

4. Colanero, S., Perata, P. \& Gonzali, S. What's behind purple tomatoes? Insight into the mechanisms of anthocyanin synthesis in tomato fruits. Plant Physiol. 182, 1841-1853 (2020).

5. Mori, S., Kobayashi, H., Hoshi, Y., Kondo, M. \& Nakano, M. Heterologous expression of the flavonoid 3'5'-hydroxylase gene of Vinca major alters flower color in transgenic Petunia hybrida. Plant Cell Rep. 22, 415-421 (2004).

6. Grotewold, E. The genetics and biochemistry of floral pigments. Annu. Rev. Plant Biol. 57, 761-780 (2006).

7. Sheehan, $\mathrm{H}$. et al. MYB-FL controls gain and loss of floral UV absorbance, a key trait affecting pollinator preference and reproductive isolation. Nat. Genet. 48, 159-166 (2016)

8. Davies, K. M. et al. Enhancing anthocyanin production by altering competition for substrate between flavonol synthase and dihydroflavonol 4-reductase. Euphytica 131, 259-268 (2003).

9. Shirley, B. W., Hanley, S. \& Goodman, H. M. Effects of ionizing radiation on a plant genome: analysis of two Arabidopsis transparent testa mutations. Plant Cell 4, 333-347 (1992).

10. Sun, W. et al. Molecular and biochemical analysis of chalcone synthase from Freesia hybrid in flavonoid biosynthetic pathway. PLOS ONE 10, e0119054 (2015).

11. Yuan, Y. W., Rebocho, A. B., Sagawa, J. M., Stanley, L. E. \& Bradshaw, H. D. Competition between anthocyanin and flavonol biosynthesis produces spatial pattern variation of floral pigments between Mimulus species. Proc. Natl Acad. Sci. USA 113, 2448-2453 (2016).

12. Dare, A. P. et al. Phenotypic changes associated with RNA interference silencing of chalcone synthase in apple (Malus $\times$ domestica). Plant J. $\mathbf{7 4}$ 398-410 (2013)

13. Dare, A. P. et al. Silencing a phloretin-specific glycosyltransferase perturbs both general phenylpropanoid biosynthesis and plant development. Plant J. 91, 237-250 (2017).

14. Watkins, J. M., Hechler, P. J. \& Muday, G. K. Ethylene-induced flavonol accumulation in guard cells suppresses reactive oxygen species and moderates stomatal aperture. Plant Physiol. 164, 1707-1717 (2014).

15. Watkins, J. M., Chapman, J. M. \& Muday, G. K. Abscisic acid-induced reactive oxygen species are modulated by flavonols to control stomata aperture. Plant Physiol. 175, 1807-1825 (2017).

16. Buer, C. S. \& Muday, G. K. The transparent testa4 mutation prevents flavonoid synthesis and alters auxin transport and the response of Arabidopsis roots to gravity and light. Plant Cell 16, 1191-1205 (2004).

17. Buer, C. S., Kordbacheh, F., Truong, T. T., Hocart, C. H. \& Djordjevic, M. A. Alteration of flavonoid accumulation patterns in transparent testa mutants disturbs auxin transport, gravity responses, and imparts long-term effects on root and shoot architecture. Planta 238, 171-189 (2013).

18. Lewis, D. R. et al. Auxin and ethylene induce flavonol accumulation through distinct transcriptional networks. Plant Physiol. 156, 144-164 (2011).

19. Kuhn, B. et al. 7-rhamnosylated flavonols modulate homeostasis of the plant hormone auxin and affect plant development. J. Biol. Chem. 291, 5385-5395 (2016).

20. Ringli, C. et al. The modified flavonol glycosylation profile in the Arabidopsis rol1 mutants results in alterations in plant growth and cell shape formation. Plant Cell 20, 1470-1481 (2008).

21. Lan, X. et al. Flavonoids and ROS play opposing roles in mediating pollination in ornamental kale (Brassica oleracea var. acephala). Mol. Plant 10, 1361-1364 (2017).
22. Chen, J., Ullah, C., Reichelt, M., Gershenzon, J. \& Hammerbacher, A. Sclerotinia sclerotiorum circumvents flavonoid defenses by catabolizing flavonol glycosides and aglycones. Plant Physiol. 180, 1975-1987 (2019).

23. Muhlemann, J. K., Younts, T. L. \& Muday, G. K. Flavonols control pollen tube growth and integrity by regulating ROS homeostasis during high-temperature stress. Proc. Natl Acad. Sci. USA 115, E11188-E11197 (2018).

24. Yin, R. et al. Kaempferol 3-O-rhamnoside-7-O-rhamnoside is an endogenous flavonol inhibitor of polar auxin transport in Arabidopsis shoots. N. Phytol. 201, 466-475 (2014).

25. Xiao, Z. et al. Extraction, identification, and antioxidant and anticancer tests of seven dihydrochalcones from Malus 'Red Splendor' fruit. Food Chem. 231, 324-331 (2017).

26. Xiao, Z., Wang, Y., Wang, J., Li, P. \& Ma, F. Structure-antioxidant capacity relationship of dihydrochalcone compounds in. Malus. Food Chem. 275 354-360 (2019).

27. Cornille, A. et al. A multifaceted overview of apple tree domestication. Trends Plant Sci. 24, 770-782 (2019).

28. Cornille, A., Giraud, T., Smulders, M. J., Roldán-Ruiz, I. \& Gladieux, P. The domestication and evolutionary ecology of apples. Trends Genet. 30, 57-65 (2014).

29. Duan, N. et al. Genome re-sequencing reveals the history of apple and supports a two-stage model for fruit enlargement. Nat. Comm. 8, 249 (2017).

30. Espley, R. V. et al. Multiple repeats of a promoter segment causes transcription factor autoregulation in red apples. Plant Cell 21, 168-183 (2009).

31. Espley, R. V. et al. Red colouration in apple fruit is due to the activity of the MYB transcription factor, MdMYB10. Plant J. 49, 414-427 (2007)

32. Shivanna, K. R. \& Rangaswamy, N. S. (eds). Pollen Biology, a Laboratory Manual (Springer-Verlag 1992)

33. Tian, J. et al. MCMYB10 regulates coloration via activating $M C F 3^{\prime} H$ and later structural genes in ever-red leaf crabapple. Plant Biot. J. 13, 948-961 (2015).

34. Meng, D. et al. Decreased sorbitol synthesis leads to abnormal stamen development and reduced pollen tube growth via an MYB transcription factor, MdMYB39L, in apple (Malus domestica). N. Phytol. 217, 641-656 (2018).

35. Guan, Y., Guo, J., Li, H. \& Yang, Z. Signaling in pollen tube growth: crosstalk, feedback, and missing links. Mol. Plant 6, 1053-1064 (2013).

36. Takeuchi, H. \& Higashiyama, T. Tip-localized receptors control pollen tube growth and LURE sensing in Arabidopsis. Nature 531, 245-248 (2016).

37. Feng, Q. N., Liang, X., Li, S. \& Zhang, Y. The ADAPTOR PROTEIN-3 complex mediates pollen tube growth by coordinating vacuolar targeting and organization. Plant Physiol. 177, 216-225 (2018).

38. Chen, D. \& Zhao, J. Free IAA in stigmas and styles during pollen germination and pollen tube growth of Nicotiana tabacum. Physiol. Plant. 134, 202-215 (2008).

39. Wu, J. Z., Lin, Y., Zhang, X. L., Pang, D. W. \& Zhao, J. IAA stimulates pollen tube growth and mediates the modification of its wall composition and structure in Torenia fournieri. J. Exp. Bot. 59, 2529-2543 (2008).

40. Dal Bosco, C. et al. The endoplasmic reticulum localized PIN8 is a pollenspecific auxin carrier involved in intracellular auxin homeostasis. Plant J. 71, 860-870 (2012).

41. Ding, Z. et al. ER-localized auxin transporter PIN8 regulates auxin homeostasis and male gametophyte development in Arabidopsis. Nat. Comm. 3, 941 (2012).

42. Rieu, I., Twell, D. \& Firon, N. Pollen development at high temperature: from acclimation to collapse. Plant Physiol. 173, 1967-1976 (2017).

43. Qin, Y. et al. Penetration of the stigma and style elicits a novel transcriptome in pollen tubes, pointing to genes critical for growth in a pistil. PLoS Genet. 5, e1000621 (2009).

44. Vogt, T., Pollak, P., Tarlyn, N. \& Taylor, L. P. Pollination-or wound-induced kaempferol accumulation in petunia stigmas enhances seed production. Plant Cell 6, 11-23 (1994).

45. Ylstra, B. et al. Flavonols stimulate development, germination, and tube growth of tobacco pollen. Plant Physiol. 100, 902-907 (1992).

46. Kevan, P., Giurfa, M. \& Chittka, L. Why are there so many and so few white flowers? Trends Plant Sci. 1, 252 (1996).

47. Dag, A. Stern, R. A. \& Shafir, S. Honey bee (Apis mellifera) strains differ in apple (Malus domestica) pollen foraging preference. J. Apic. Res. 44, 15-20 (2005).

48. Garratt, M. P. D. et al. Apple pollination: demand depends on variety and supply depends on pollinator identity. PLOS ONE 11, e0153889 (2016).

49. Ramírez, F. \& Davenport, T. L. Apple pollination: a review. Sci. Hortic. 162 188-203 (2013). 
50. Dai, H. et al. Development of a seedling clone with high regeneration capacity and susceptibility to Agrobacterium in apple. Sci. Hortic. 164, 202-208 (2013).

51. Li, P., Ma, F. \& Cheng, L. Primary and secondary metabolism in the sunexposed peel and the shaded peel of apple fruit. Physiol. Plant. 148, 9-24 (2013).
52. Malnoy, M., Reynoird, J. P., Mourgues, F., Chevreau, E. \& Simoneau, P. A method for isolating total RNA from pear leaves. Plant Mol. Biol. Rep. 19, 69a-69f (2001).

53. Kho, Y. O. \& Baer, J. Observing pollen tubes by means of fluorescence Euphytica 17, 298-302 (1968). 